\title{
Low incidence of positive smooth muscle antibody and high incidence of isolated IgM elevation in Chinese patients with autoimmune hepatitis and primary biliary cirrhosis overlap syndrome: a retrospective study
}

Pan Zhao ${ }^{1}$ and Yukun Han ${ }^{2^{*}}$

Background: Up to now, few data are available regarding the clinical characteristics of autoimmune hepatitis and primary biliary cirrhosis overlap syndrome. The study was to investigate and analyze the prevalent and clinical features of Chinese patients with this disease.

Methods: Clinical data on patients diagnosed as autoimmune hepatitis and primary biliary cirrhosis overlap syndrome in our hospital from January 2001 to December 2006 were collected and analyzed.

Results: Overlap syndrome of autoimmune hepatitis and primary biliary cirrhosis accounted for $10.33 \%$ of patients with autoimmune liver diseases during the past six years. For these patients with overlap syndrome, xanthochromia, lethargy and anorexia were the predominant complaints; a low incidence (14/146) of smooth muscle antibody positivity and a high incidence (37/89) of isolated IgM elevation were the main serological characteristics.

Conclusions: Overlap syndrome of autoimmune hepatitis and primary biliary cirrhosis was not rare in Chinese patients with clinical manifests of autoimmune liver diseases. Overlap of the diseases should not be disregarded when isolated IgM elevation was exhibited, and smooth muscle antibody might have little diagnostic significance in the overlap syndrome. If it was difficult to make a definite diagnosis, liver biopsy was necessary.

Keywords: autoantibody, overlap syndrome, autoimmune hepatitis, primary biliary cirrhosis

\section{Background}

Autoimmune hepatitis (AIH), primary biliary cirrhosis (PBC), and primary sclerosing cholangitis (PSC) are three major clinicopathologic entities of autoimmune liver diseases. Besides, some patients with autoimmune liver disease present with characteristics of a second autoimmune liver disease (i.e. AIH and PBC overlapping). These cases have been defined as overlap syndrome. Patients with overlap syndrome usually present with nonspecific symptoms, including lethargy, arthralgias, and myalgias. A combination of clinical and laboratory or pathologic criteria is necessary for the diagnosis of the disease. In the laboratory

\footnotetext{
* Correspondence: yukunh@yahoo.com.cn

${ }^{2}$ Case-history Office, Beijing 302 Hospital, Beijing 100039, China

Full list of author information is available at the end of the article
}

test, autoantibodies are the serological hallmarks. Serum antinuclear antibody (ANA), smooth muscle antibody (SMA) and antimitochondrial antibody (AMA) are routinely detected in these patients [1].

Although combined features of both PBC and PSC have been reported in single cases, there is no clear evidence for the existence of an overlap of PBC and PSC [2]. The overlap syndrome of $\mathrm{AIH}$ and $\mathrm{PBC}$ is the most common form, and it shows a more progressive course towards liver cirrhosis and liver failure than AIH or PBC alone [3-5]. However, up to now, the pathogenesis of overlap syndrome is poorly understood [6], and few data are available regarding the clinical characteristics of this disease, and moreover, reports on the prevalent and serological features of this condition in Chinese population 
are still lacking. The aim of this retrospective study is to investigate and analyze the prevalent and clinical features of Chinese patients with $\mathrm{AIH}$ and $\mathrm{PBC}$ overlap syndrome.

\section{Methods}

\subsection{Patients}

Patients diagnosed as overlap syndrome of AIH and PBC in 302 hospital from January 2001 to December 2006 were included in the retrospective study. The diagnosis was based on the criteria established by Chazouillères $\mathrm{O}$, et al [7]. Exclusion criteria included coinfection with hepatitis A, C, D, E, Epstein-Barr virus, cytomegalovirus or HIV; the presence of other forms of liver diseases such as alcoholic liver disease, drug hepatitis or Wilson's disease. 146 patients were eventually enrolled in our study. Informed written consent for the analysis was obtained from each patient. The study was approved by the ethics committee of Beijing 302 Hospital.

\subsection{Serological markers and liver histopathology}

Serum autoantibodies, including antinuclear antibody (ANA), smooth muscle antibody (SMA) and antimitochondrial antibody (AMA) were tested using indirect immunofluorescence with the standard methods (Euroimmun Medizinnische Labordiagnostika AG, Germany), and sera were considered to be positive when they produced a reaction at a dilution of $\geq 1: 100$. Immunoglobulin (Ig) assay were taken with the mothod of immunological turbidimetry (Diasys Diagnostic Systems, China). The normalized levels of IgG, IgM and IgA were respectively 7.23-16.6 g/L, 0.63-2.77 g/L and 0.69-3.82 g/L.

Biochemical profiles, including alanine transarninase (ALT), aspartate aminotransferase (AST), total bilirubin (TBil), gamma glutamyl transferase (GGT) and alkaline phosphatase (ALP) were measured using standard laboratory procedure. The normalized levels of ALT, AST, TBil, GGT and ALP were respectively $<40 \mathrm{U} / \mathrm{L},<40 \mathrm{U} / \mathrm{L}$, $<17.1 \mathrm{umol} / \mathrm{L}, 7-32 \mathrm{U} / \mathrm{L}$, and 40-150 U/L.

Liver biopsy was performed in some cases for definite diagnosis, and biopsy specimens were examined in the Pathology Department.

\subsection{Statistical analysis}

Data analysis were performed using SAS 9.2 software (SAS Institute Inc., Cary, NC, USA) and the quantitative data were expressed as mean and standard deviation.

\section{Results}

\subsection{Baseline characteristics and prevalent features}

In this six-year retrospective survey based on our hospital, a total of 1413 patients was diagnosed as autoimmune liver diseases, of which, 577 were $\mathrm{AIH}, 685$ were $\mathrm{PBC}, 5$ were PSC, 146 were overlap syndrome of AIH and $\mathrm{PBC}$, and none were overlap syndrome of $\mathrm{AIH}$ and
PSC. Overlap syndrome of $\mathrm{AIH}$ and $\mathrm{PBC}$ accounts for $10.33 \%$ of patients with autoimmune liver diseases, and this proportion was similar to that in previous reports in India and France [8,9]. The characteristics of the 146 patients at the diagnosis of overlap syndrome of $\mathrm{AIH}$ and $\mathrm{PBC}$ were described in Table 1.

\subsection{Clinical features}

We viewed the 146 case histories, and found that, as the chief complaints at the diagnosis, xanthochromia occurs in 86 patients, with the incidence of $58.90 \%$; lethargy occurs in 79 patients, with the incidence of $54.11 \%$; anorexia occurs in 75 patients, with the incidence of $51.37 \%$; pruritus occurs in 51 patients, with the incidence of $34.93 \%$; discomfort in hepatic region occurs in 33 patients, with the incidence of $22.60 \%$; arthralgias or myalgias occurs in 33 patients, with the incidence of $22.60 \%$; asymptomatic elevation of serum liver enzymes occurs in 20 patients, with the incidence of $13.70 \%$; splenomegaly occurs in 12 patients, with the incidence of $8.22 \%$. Details were summarized in Table 2 .

Of the 146 patients, 140 had positive ANA, accounting for $95.89 \%$; 129 had positive AMA, accounting for 88.36\%; 14 had positive SMA, accounting for $9.59 \%$. Details were seen in Table 3.

A total of 89 patients with immunoglobulin assay was identified. Of these patients, 37 had isolated IgM elevation, accounting for $41.57 \%$; 17 had isolated IgG elevation, accounting for 19.10\%; 23 had both IgM and IgG elevation, accounting for 25.84\%; 12 had neither IgM nor IgG elevation, accounting for $13.48 \%$. The results were summarized in Table 4.

58 patients underwent liver biopsy. Diagnostic pathological changes, including bile duct lesion, interface hepatitis and plasma cell infiltration were observed in all patients. Summarization of the pathological features for these patients was seen in Yanling Sun's previous study

Table 1 Characteristics of patients at the diagnosis of AIH and PBC overlap syndrome

\begin{tabular}{ll}
\hline & Value \\
\hline sex: female/male & $125 / 21$ \\
age (years) & $46.50 \pm 10.08$ \\
TBil (umol/L) & $43.08 \pm 27.94$ \\
ALT (U/L) & $116.80 \pm 37.10$ \\
AST (U/L) & $77.02 \pm 32.75$ \\
GGT (U/L) & $369.12 \pm 329.32$ \\
ALP (U/L) & $399.40 \pm 221.53$ \\
$\operatorname{lgM}$ & $3.91 \pm 2.08$ \\
$\operatorname{lgG}$ & $18.79 \pm 1.56$ \\
& $n=146$ \\
\hline
\end{tabular}

ALT, alanine transarninase; AST, aspartate aminotransferase; TBil, total bilirubin; GGT, gamma glutamyl transferase; ALP, alkaline phosphatase; Ig, immunoglobulin. 
Table 2 Chief complaints of patients at the diagnosis of AIH and PBC overlap syndrome

\begin{tabular}{lll}
\hline Chief complaint & Case & Incidence (\%) \\
\hline Xanthochromia & 86 & 58.90 \\
Lethargy & 79 & 54.11 \\
Anorexia & 75 & 51.37 \\
Pruritus & 51 & 34.93 \\
discomfort in hepatic region & 33 & 22.60 \\
arthralgias or myalgiasg & 33 & 22.60 \\
asymptomatic elevation of serum liver & 20 & 13.70 \\
enzymes & \multicolumn{2}{|c}{8.22} \\
splenomegaly & 12 & \\
& $\mathrm{n}=146$ & \\
\hline
\end{tabular}

[10]. Pathological changes in a patient with AIH and PBC overlap syndrome were shown in Figure 1.

\section{Discussion}

The worldwide prevalence of overlap syndrome of AIH and $\mathrm{PBC}$ is unknown. The first cases of this disease were reported almost 30 years ago, and this entity was assumed to be rare [11]. However, in our hospital, $10.33 \%$ of patients with autoimmune liver diseases were diagnosed as overlap syndrome of AIH and PBC based on the clinical manifests, laboratory test, and liver biopsy during the six years. Our study also showed that, similar to other studies $[12,13]$, overlap syndrome of AIH and PBC was more common in female patients, with a female:male ratio of roughly $6: 1$. However, the median age of patients with overlap syndrome was older than that of a previous report about European patients [9].

Presentation of autoimmune liver diseases varies widely, ranging from asymptomatic elevations of serum liver enzymes to massive hepatic necrosis resulting in fulminant hepatic failure, and there are no disease-specific clinical features. Günsar F, et al [14] reported that, lethargy was the most common symptom in these patients. Our study showed that xanthochromia, lethargy and anorexia were the predominant three chief complaints, that was, besides lethargy, xanthochromia was also a most common symptom in Chinese patients with overlap syndrome of AIH and $\mathrm{PBC}$.

Table 3 Features of autoantibodies in patients

\begin{tabular}{lll}
\hline Features & No. & Incidence (\%) \\
\hline ANA positive & 140 & 95.89 \\
AMA positive & 129 & 88.36 \\
SMA positive & 14 & 9.59 \\
both ANA and AMA positive & 122 & 83.56 \\
both SMA and AMA positive & 10 & 6.85 \\
& $\mathrm{n}=146$ & \\
\hline
\end{tabular}

ANA, antinuclear antibody; AMA, antimitochondrial antibody; SMA, smooth muscle antibody.
Table 4 Features of immunoglobulin (Ig) elevation in patients

\begin{tabular}{lll}
\hline Features & No. & Percentage (\%) \\
\hline isolated IgM elevation & 37 & 41.57 \\
isolated IgG elevation & 17 & 19.10 \\
both IgM and IgG elevation & 23 & 25.84 \\
neither IgM nor IgG elevation & 12 & 13.48 \\
\cline { 2 - 3 } Total & 89 & 100.00 \\
\hline
\end{tabular}

Serum autoantibodies have steadily established themselves as critical biomarkers for the diagnosis of autoimmune diseases [15]. For the North-American and European population, ANA and SMA constitute the standard repertoire for the diagnosis of autoimmune hepatitis, and AMA is the diagnostic marker of primary biliary cirrhosis $[7,16]$. In our study, the majority of patients had positive serum ANA (140/146) and AMA (129/146), whereas only minor patients $(14 / 146)$ had positive serum SMA. This result showed that, there was a low incidence of positive serum SMA in Chinese patients with overlap syndrome of AIH and $\mathrm{PBC}$, which might indicate that serum SMA may have little diagnostic significance in the overlap syndrome for Chinese patients. This finding agreed with another investigation performed by Zhenxia Liu [17].

Some studies had shown that the serum immunoglobulins could elevate in most cases of autoimmune liver diseases [18-22] and types of the elevated immunoglobulins were distinctive in different categories of autoimmune liver diseases. IgG was the predominant immunoglobulin elevated in serum of AIH patients while IgM was elevated in most patients with PBC [23-25]. However, in our study, isolated IgM elevation was preponderant (37/89), which presented with features of PBC. Previously, a German

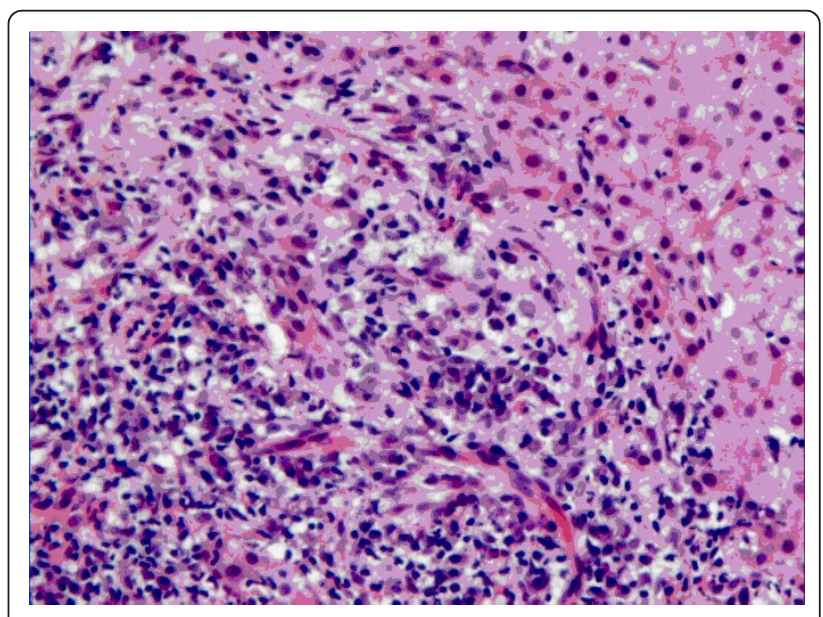

Figure 1 Typical pathological changes (bile duct lesion, interface hepatitis and plasma cell infiltration) in a Chinese patient diagnosed as AIH and PBC overlap syndrome. (HE, $\times 200)$. 
study showed that patients with overlap syndrome of AIH and $\mathrm{PBC}$ presented with typical features of $\mathrm{PBC}$ when compared to $\mathrm{AIH}$ and $\mathrm{PBC}$ patients [13]. The reason, we inferred, might be that serological markers were often featured with one predominant pathological change to the other in overlap syndrome. But how to distinguish the predominant one needed further studies.

\section{Conclusion}

Overlap syndrome of autoimmune hepatitis and primary biliary cirrhosis was not rare in Chinese patients with clinical manifests of autoimmune liver diseases. Overlap of the diseases should not be disregarded when isolated IgM elevation was exhibited, and smooth muscle antibody might have little diagnostic significance in the overlap syndrome. If it was difficult to make a definite diagnosis, liver biopsy was necessary.

\section{List of abbreviations}

AlH: autoimmune hepatitis; ALP: alkaline phosphatase; ALT: alanine transarninase; AMA: antimitochondrial antibody; ANA: antinuclear antibody; AST: aspartate aminotransferase; GGT: gamma glutamyl transferase; PBC: primary biliary cirrhosis; PSC: primary sclerosing cholangitis; SMA: antismooth muscle antibody; Tbil: total bilirubin.

\section{Acknowledgements and Funding}

We are grateful to Yanling Sun for her assistance in supplying the pathological picture.

No funding was provided to this study.

\section{Author details}

'Liver Failure Therapy and Research Center, Beijing 302 Hospital, Beijing 100039, China. ${ }^{2}$ Case-history Office, Beijing 302 Hospital, Beijing 100039, China.

\section{Authors' contributions}

$\mathrm{YH}$ designed the study; PZ analyzed the data and wrote the manuscript. PZ and $\mathrm{YH}$ were both involved in the acquisition of the data. All authors read and approved the final manuscript.

\section{Competing interests}

The authors declare that they have no competing interests.

Received: 20 August 2011 Accepted: 3 January 2012

Published: 3 January 2012

\section{References}

1. Björnsson E, Talwalkar J, Treeprasertsuk S, Neuhauser M, Lindor K: Patients with typical laboratory features of autoimmune hepatitis rarely need a liver biopsy for diagnosis. Clin Gastroenterol Hepatol 2011, 9(1):57-63.

2. Rust $C$, Beuers U: Overlap syndromes among autoimmune liver diseases. World J Gastroenterol 2008, 14(21):3368-3373.

3. Hirschfield GM, Al-Harthi N, Heathcote EJ: Current status of therapy in autoimmune liver disease. Therap Adv Gastroenterol 2009, 2(1):11-28.

4. Coss Adame E, Granados J, Uribe M, Torre A: Does HLA-DR7 differentiate the overlap syndrome of auto-immune hepatitis-primary biliary cirrhosis (AlH-PBC) from those with auto-immune hepatitis type 1? Ann Hepatol 2011, 10(1):28-32.

5. Cooper GS, Bynum ML, Somers EC: improved prevalence estimates and understanding of clustering of diseases. J Autoimmun 2009, 33(3-4):197-207.

6. Czaja AJ: Autoantibodies as prognostic markers in autoimmune liver disease. Dig Dis Sci 2010, 55:2144-2161.

7. Chazouillères $\mathrm{O}$, Wendum $\mathrm{D}$, Serfaty $\mathrm{L}$, Montembault $\mathrm{S}$, Rosmorduc $\mathrm{O}$, Poupon R: Primary biliary cirrhosis-autoimmune hepatitis overlap syndrome: clinical features and response to therapy. Hepatology 1998 28(2):296-301.

8. Arulprakash S, Sasi AD, Bala MR, Pugazhendhi T, Kumar SJ: Overlap syndrome: autoimmune hepatitis with primary biliary cirrhosis. J Assoc Physicians India 2010, 58:455-456

9. Heurgué A, Vitry F, Diebold MD, Yaziji N, Bernard-Chabert B, Pennaforte IL, Picot R, Louvet H, Frémond L, Geoffroy P, Schmit JL, Cadiot G, Thiéfin G: Overlap syndrome of primary biliary cirrhosis and autoimmune hepatitis: a retrospective study of 115 cases of autoimmune liver disease. Gastroenterol Clin Biol 2007, 31(1):17-25.

10. Sun YL, Zhao JM, Meng X, Li WS: Clinical and pathological studies on the autoimmune hepatitis and primary biliary cirrhosis overlap syndrome. Med J Chin PLA 2006, 31(7):662-665, (in Chinese).

11. Beuers U: Hepatic overlap syndromes. J Hepatol 2005, 42:593-599.

12. Czaja AJ: Autoimmune liver disease. Curr Opin Gastroenterol 2008, 24(3):298-305

13. Lohse AW, zum Büschenfelde KH, Franz B, Kanzler S, Gerken G, Dienes HP: Characterization of the overlap syndrome of primary biliary cirrhosis (PBC) and autoimmune hepatitis: evidence for it being a hepatitic form of PBC in genetically susceptible individuals. Hepatology 1999, 29(4):1078-1084.

14. Günsar F, Akarca US, Ersöz G, Karasu Z, Yüce G, Batur Y: Clinical and biochemical features and therapy responses in primary biliary cirrhosis and primary biliary cirrhosis-autoimmune hepatitis overlap syndrome. Hepatogastroenterology 2002, 49(47):1195-1200

15. Selmi C, Gershwin ME: Autoantibodies in autoimmune liver disease: biomarkers versus epiphenomena. Gut 2010, 59(6):712-713.

16. Neuhauser M, Bjornsson E, Treeprasertsuk S, Enders F, Silveira M, Talwalkar J, Lindor K: Autoimmune hepatitis-PBC overlap syndrome: a simplified scoring system may assist in the diagnosis. Am J Gastroenterol 2010, 105(2):345-353.

17. Liu ZX, Liu Y, Huang XJ, Liu YL: Clinical studies on AlH, PBC and AlH-PBC overlap syndrome. Hebei Med J 2011, 33(1):49-50, (in Chinese).

18. Teufel A, Weinmann A, Kahaly GJ, Centner C, Piendl A, Wörns M, Lohse AW Galle PR, Kanzler S: Concurrent autoimmune diseases in patients with autoimmune hepatitis. J Clin Gastroenterol 2010, 44(3):208-213.

19. Jalihal $\mathrm{A}$, Telisinghe $\mathrm{PU}$, Chong $\mathrm{VH}$ : Profiles of autoimmune hepatitis in Brunei Darussalam. Hepatobiliary Pancreat Dis Int 2009, 8(6):602-607.

20. Moreira RK, Revetta F, Koehler E, Washington MK: Diagnostic utility of IgG and IgM immunohistochemistry in autoimmune liver disease. World $\rfloor$ Gastroenterol 2010, 16(4):453-7.

21. Kawa S, Kitahara K, Hamano H, Ozaki Y, Arakura N, Yoshizawa K, Umemura T, Ota M, Mizoguchi S, Shimozuru Y, Bahram S: A novel immunoglobulin-immunoglobulin interaction in autoimmunity. PLOS One 2008, 3(2):e1637

22. Washington MK Autoimmune liver disease: overlap and outliers. Mod Pathol 2007, 20(Suppl 1):S15-S30.

23. Cabibi D, Tarantino G, Barbaria F, Campione M, Craxì A, Di Marco V: Intrahepatic lgG/lgM plasma cells ratio helps in classifying autoimmune liver diseases. Dig Liver Dis 2010, 42(8):585-592.

24. Lee $H$, Stapp RT, Ormsby AH, Shah W: The usefulness of IgG and IgM immunostaining of periportal inflammatory cells (plasma cells and lymphocytes) for the distinction of autoimmune hepatitis and primary biliary cirrhosis and their staining pattern in autoimmune hepatitisprimary biliary cirrhosis overlap syndrome. Am J Clin Pathol 2010, 133(3):430-437.

25. Krawitt EL: Discrimination of autoimmune hepatitis: autoantibody typing and beyond. J Gastroenterol 2011, 46(Suppl 1):39-41.

\section{Pre-publication history}

The pre-publication history for this paper can be accessed here: http://www.biomedcentral.com/1471-230X/12/1/prepub

\section{doi:10.1186/1471-230X-12-1}

Cite this article as: Zhao and Han: Low incidence of positive smooth muscle antibody and high incidence of isolated lgM elevation in Chinese patients with autoimmune hepatitis and primary biliary cirrhosis overlap syndrome: a retrospective study. BMC Gastroenterology 2012 12:1. 